\title{
MOCVD 反应器中流场和热场的数值研究
}

\author{
钟树泉, 任晓敏, 黄永清, 王琦, 黄辉 \\ 北京邮电大学光通信与光波导教育部重点实验室, 北京 100876 \\ E-mail: shuqzhong@gmail.com
}

2009-03-02 收稿, 2009-08-11 接受

国家重点基础研究发展计划(编号: 2003CB314901)、教育部长江学者和创新团队发展计划(编号: IRT0609)、国家高技术研究发展计划(编号: 2006AA03Z416，2007AA03Z418)、国家自然科学基金(批准号：10875018,10773002，60576018)、国家自然科学基金重点项目(批准号: 90601002)和高等学校学科创新引智计划(编号: B07005)资助

摘要 利用二维动力学模型, 通过变化 MOCVD (metal organic chemical vapor deposition) 反应器的进气流量、操作压力、补底温度、基座旋转等几个重要工艺控制参 数, 计算了反应器内部均匀的流场和热场分布的形态变化, 描述了输运过程中产生的多 种流动现象, 并给出了相应的分析与说明. 在此基础上, 通过微扰反应器的进气量, 计 算并图形化了质量输运过程的瞬态行为, 分析了延迟时间、驰豫振荡、自脉动振荡等瞬 态现象产生的原因, 为高品质外延生长工艺的设计与实施, 提供了有益的解决途径.

关键词

晶体外延生长

MOCVD 反应器

输运过程

热流场
MOCVD 是由美国的 Manasevit 等人 11 于 1968 年 首先提出的批量制备化合物半导体材料的技术. 目 前, 该设备所生产的微电子、光电子材料和器件, 已 成为提高国防武器与指挥系统性能的关键技术, 且 成为高速光通信、3G 移动通信、宽带卫星通信、高 效太阳能电池、宇宙深层空间探测技术之基石 ${ }^{[2 \sim 6]}$.

在 MOCVD 反应器中外延异质结构的材料时, 用来生长晶体的各组份和掺杂剂, 都是以气态方式 进人反应器的; 该系统通过周期性地切换各种反应 气体控制其流人量, 在衬底上外延出特定组份、特定 厚度、特定导电类型、特定电学和光学性能的晶体材 料. 然而, 周期性地切换控制前驱物进入反应器, 到 衬底上方的反应物形成稳定的流动状态, 存在着许 多瞬态过程. 特别是基于量子点、量子线、量子阱超 晶格等纳米材料的制备时, 其外延生长过程更为复 杂, 瞬态现象更是随处可见.

自 1980 以来, 国内外科技人员利用水力学实验、 光学测量 ${ }^{[7 \sim 9]}$ 和数值计算 ${ }^{[10 \sim 20]}$ 等方法, 对 MOCVD 反
应器内部极端复杂的质量输运过程进行了大量的研 究, 取得了许多有价值的成果, 并且在 1990 年左右, 形成研究的第一个高潮. 随后, 其研究工作呈现持续 增长的态势; 近几年来, 这一课题发表的研究成果颇 多, 并形成该领域研究的第二个高潮. 但是, 以上研 究内容还主要集中于稳态方面, 侧重于新材料的创 新、新结构的开发以及新型器件的制备. 对于有价值 的瞬态特性的研究, 在国际上还鲜见报道; 而瞬态行 为是客观存在的现象, 它对化合物半导体材料纳米 级多层结构的外延生长, 是不容忽视的要素.

本文以英国 THOMAS SWAN 公司 $3 \times 2$ " CCS LP-MOCVD 设备为研究对象, 首先从 MOCVD 反应 器的稳态研究出发, 大范围变化工艺参数, 考察其流 体力学特征, 并提取设备工艺参数进行优化计算, 得 出了反应器中热流场稳态分布变化的规律. 基于以 上计算与分析, 通过微扰反应器的进气量, 进一步考 察反应器内气体流动的瞬态行为, 计算得到了一些 有价值的新成果, 从宏观上较全面地把握了反应器 
内部流体力学的输运特征.

\section{1 数学物理模型及计算条件}

\subsection{MOCVD 反应器几何结构}

如图 1 所示, MOCVD 反应器为圆柱腔结构, 几 何尺寸为基座半径 $R=70 \mathrm{~mm}$, 顶部 Showerhead 与基 座的距离(反应器的高度) $h=10 \mathrm{~mm}$, 腔体高度 $H=300$ $\mathrm{mm}$, 尾气流道的宽度 $D=12 \mathrm{~mm}$. Showerhead 为气体 分布器, 它是开有很多小孔的一块平板, 每平方英寸 约有 100 个小孔, 其大小和基座相当, 以确保气孔完 全覆盖整个基座; 另外, Showerhead 可被冷却水控温 在 $50 \sim 60^{\circ} \mathrm{C}$. Showerhead 采用如此复杂结构之目的, 是为了防止III、V 族源的预混反应. 基座是由石墨材 料制成, 衬底可放置在基座上的凹槽中; 基座具有加 热功能, 可使衬底升温至 $1250 \mathrm{~K}$; 在材料生长的时候, 基座连同其上的衬底是旋转的, 转速为 $0 \sim 800 \mathrm{r} / \mathrm{min}$. 反应器的内外侧壁皆为石英材料, 外侧壁用冷却水 制冷, 以保证其温度接近室温.

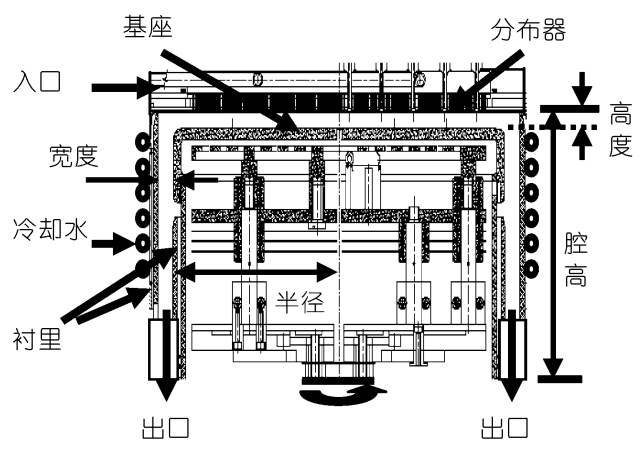

图 1 垂直式 MOCVD 反应器简图

\section{2 数学物理模型}

MOCVD 反应器的载气一般为 $\mathrm{H}_{2}$, 它携带III族 MO 源和 V 族源从 Showerhead 的小孔, 均匀喷人反 应器; 混合气体流经被适当加热的祄底后, 发生化学 反应并产生单体分子; 这些单体分子将会碰撞、凝 聚、结晶、生长而成为分子团粒子, 最终在衬底表面 上形成异质结构薄层材料. 流体中未沉积在衬底的 分子团, 将随反应物、副产物及载气, 经过侧壁尾气 流道离开反应器, 经尾气处理系统后排放.

由于半导体材料的外延生长工艺, 通常发生在 700 1200 K, 6.66 101.3 kPa 条件下, 反应器内部气 体流动速度很低, 可以认为是不可压、连续、理想流
体; 其流体力学特征满足质量、动量、能量和组分守 恒等方程, 可由一组微分方程表示为:

质量守恒方程: $\quad \frac{\mathrm{d} \rho}{\mathrm{d} t}=-\rho \nabla \cdot \boldsymbol{u}$,

动量守恒方程: $\rho \frac{\mathrm{d} \boldsymbol{u}}{\mathrm{d} t}=-\nabla p+\nabla \cdot \boldsymbol{\tau}+\rho \boldsymbol{f}$ ，

能量守恒方程: $\rho \frac{\mathrm{d} E}{\mathrm{~d} t}=-p \nabla \cdot \boldsymbol{u}-\nabla \cdot \boldsymbol{q}+\boldsymbol{\tau} \otimes \nabla \boldsymbol{u}+Q$,

组分守恒方程: $\frac{\partial}{\partial t}\left(\rho Y_{i}\right)+\nabla \cdot\left(\rho \boldsymbol{u} Y_{i}\right)=-\nabla \cdot \boldsymbol{J}_{i}+S_{i}$,

理想气体状态方程: $p=\rho R T / M_{\mu}$,

理想气体内能公式: $\mathrm{d} E=C_{V} \mathrm{~d} T$ ，

傅里叶热传导公式: $\boldsymbol{q}=-K \nabla T$,

式中 $\rho$ 为流体密度, $\boldsymbol{u}$ 为流体流速矢量, $t$ 为时间; $f$ 为单位质量力, $p$ 是流体压力, $\tau$ 是切应力张量; $E$ 为单位质量流体所具内能; $Q$ 为单位体积流体热 量的增量, $\boldsymbol{q}$ 为热通量向量, $\otimes$ 表示两个并矢张量的 缩并; 第 $i$ 种物质的质量分数 $Y_{i}$, 扩散通量矢量 $\boldsymbol{J}_{i}$, 化学反应在单位体积内产生的质量速率 $S_{i} ; T$ 为绝 对温度, $R$ 为气体常数, $M_{\mu}$ 为载气的分子量; $C_{v}$ 为 定容比热容; $K$ 为热导率.

\section{3 数值计算方法与计算条件}

MOCVD 反应器内部气体的流动, 不仅存在由重 力、密度差、压差引起的自然对流, 以及基座旋转引 起的强制对流; 而且还存在温度梯度引起的动量、热 量、质量传递等现象. 因此, 在不影响主要结论的前 提下, 我们作如下假设:

(i) 鉴于半导体材料的生长, 是在质量输运控制 下的连续稳态过程, 故稳态计算时可忽略时间项.

(ii) III- $V$ 族源占载气的比例不足 $1 \%$, 可忽略其 化学反应所产生的影响; 混合气体的物化性质由 $\mathrm{H}_{2}$ 决定. 由于 $\mathrm{H}_{2}$ 对于热辐射是透明的, 可忽略其热辐 射效应.

(iii) 由于反应器是轴对称几何结构, 边界条件 也是轴对称的, 数学模型可简化为二维轴对称模型.

(iv) 流体与反应器内所有壁面的作用, 均采用 无滑移边界条件. 
(v) 基座和衬底的温度可视为均匀等温, 其余各 壁面的温度也可认为满足等温或绝热条件.

以生长 $\mathrm{InP}$ 外延层为例：载气为 $\mathrm{H}_{2}, \mathrm{~V}$ 族源为磷 烷 $\left(\mathrm{PH}_{3}\right)$, III族源为三甲基铟(TMIn), TMIn 摩尔浓度 为 $3 \times 10^{-4}, \mathrm{~V} / \mathrm{III}$ 的摩尔比为 70 . 为了更有效地研究 反应器内热流场的变化规律, 我们选择一组基准工 艺条件: $h=10 \mathrm{~mm}, Q=10 \mathrm{~L} / \mathrm{min}, P=13.3 \mathrm{kPa}, \omega=$ $0 \mathrm{r} / \mathrm{min}$, 气体进口为常压 $300 \mathrm{~K}$, Showerhead 的温度 为 $350 \mathrm{~K}$, 基座表面温度 $t=923 \mathrm{~K}$, 内侧壁温度为绝 热, 外侧壁温度为绝热, $g=9.81 \mathrm{~m} / \mathrm{s}^{2}$, 腔体的计算高 度取 $H^{\prime}=30 \mathrm{~mm}$. 在求解微分方程组时, 利用了 Patankar 及 Spalding 于 1972 年所提出的 SIMPLE (semi-implicit pressure linked equation)算法, 它属于 压力修正法, 主要用于求解不可压流体. 在数值计算 中, 采用有限体积方法 FVM (finite volume method), 对单一控制体积内的物理量进行区域离散化处理. 为了适应复杂的几何边界条件, 对反应器模型不同 区域进行适当的非结构网格划分设计, 再结合各边 界条件进行计算, 并定义残差值为 $10^{-3}$ 作为收玫判据. 由于流场和温度场具有轴对称性, 所以只需给出半 个区域的计算结果.

\section{MOCVD 反应器热流场的计算与分析}

MOCVD 外延生长技术考虑的重要问题为: 较高 的晶体生长速率, 均匀的组分、掺杂与厚度, 陡峭的 异质界面分布, 同时各类源材料有较高的利用率. 为 了实现这些目标, 需要十分清晰地了解 MOCVD 反 应器内部的反应物的流动状态, 以利于在衬底附近
形成均匀的温度场和流场, 这需要很好地控制沉积 表面的反应物的输运状态

\section{1 进口乞体流量对热流场分布的影响}

在研究 MOCVD 反应器内部流场时, 采用欧拉 法的流线图, 可直观地描述其连续流体的运动形态. 如图 2(a)所示流场, 气体从垂直向下转为水平径向流 动后, 流场结构较复杂, 在反应器衬底上方空间和壁 面弯折处形成了几个对流浴旋. 对流浴旋的存在, 使 得衬底附近的反应剂分子得不到有效的更新, 由此 产生的“记忆效应”造成材料生长的不均匀现象, 即 对沉积质量产生不良的影响. 而在图 2(b)和(c)中, 反 应器内流场中的对流浴旋较少, 主流道中对流浴旋 基本消失, 在几何中心部位的较弱涡旋及在拐角弯 折处的浴旋, 对外延质量影响不大, 这样的流动条件, 较有利于晶体外延生长的均匀性. 从图 2(b)和(c)分析, 随着进气流量的增加, 当 $Q$ 大于 $10 \mathrm{~L} / \mathrm{min}$ 时, 主流道 中流体流动比较平稳.

由于温度对于化学反应极为重要, 所以生长大面 积厚度均匀的高质量晶体材料, 依赖于合理的温度场 分布状态. 如图 3(a),(b)和(c)温度场(以等温线表示, 单位: K) 所示, 随着进气流量的增加, $808.4 \mathrm{~K}$ 的等温 线由隆起逐渐转为平直, 其温度场的分布更趋均匀合 理, 这将提高半导体材料生长的均匀性. 这表明适当 增加进气量对主流道的温度场分布有明显的改善.

从图 2,3所示的流场与温度场分析发现, MOCVD 合理的进气流量可以非常有效地控制温度场的分布, 并有效地抑制对流涡旋的发生.
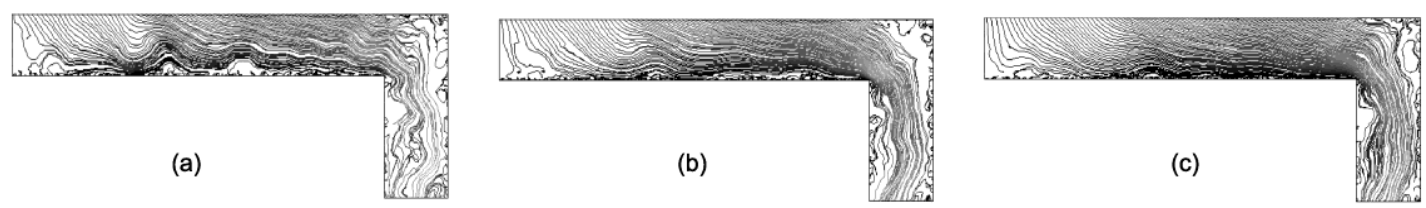

图 2 进气流量对流场结构的影响

(a) $Q=8 \mathrm{~L} / \mathrm{min}$; (b) $Q=10 \mathrm{~L} / \mathrm{min}$; (c) $Q=15 \mathrm{~L} / \mathrm{min}$
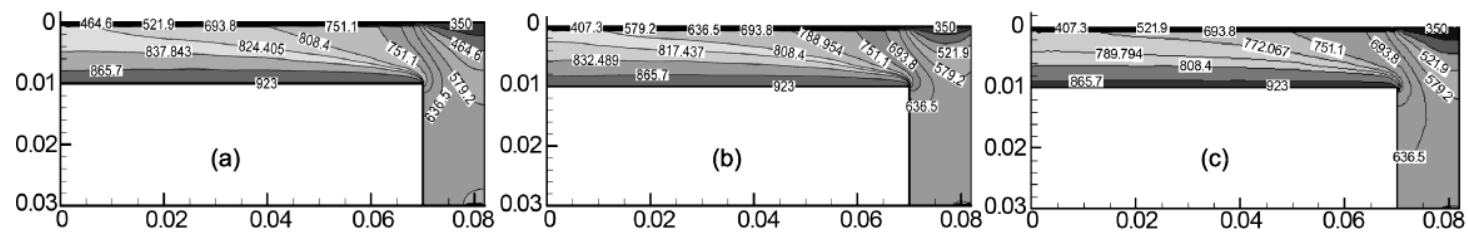

图 3 进气流量对温度场分布的影响

(a) $Q=8 \mathrm{~L} / \mathrm{min}$; (b) $Q=10 \mathrm{~L} / \mathrm{min}$; (c) $Q=15 \mathrm{~L} / \mathrm{min}$ 


\section{2 操作压力对热流场分布的影响}

图 4(a), (b) 和(c)是在其他条件不变时, 操作压力 分别为 $101.3,13.3$ 和 $6.66 \mathrm{kPa}$ 时流场分布变化图. 可 以发现随着压力的逐渐减小, 使得对流涡旋的强度 也逐渐减少, 直到使它们最后基本消失. 图 4(a)表示 在常压下的流场分布, 反应器内部流场复杂, 存在 许多浴旋并被限制在主流道处, 这将导致沿着气流 方向组分浓度剧烈的变化, 使沉积厚度变得不均匀, 这就是绝大多数 MOCVD 反应器采取低压操作的主 要原因.

在流量固定的条件下, 减小反应器内的压力, 使 得扩散系数增加, 从而导致传质系数也增大, 这有利 于反应剂分子在系统内部快速弥散, 从而减小了涡 旋再循环的强度, 有利于外延大面积厚度均匀的材 料. 但是在实际生长中, 并非压力越低越好, 在压力 太低的情况下, 系统中气体流速过大, 大部分反应剂 由泵排出, 原材料的消耗量也增加.

当其他条件不变时, 计算了 3 种操作压力的热 场情况, 发现反应器内的温度场分布的变化极小, 3 种形态都与图 3(b)的区别甚微, 所以其计算图形在 此没有列出. 这表明在特定的设备结构尺寸和一定 的工艺条件下, 操作压力的改变对温度场分布的影 响不显著.

\section{3 衬底温度对热流场分布的影响}

图 5(a), (b) 和(c)是在其他基准条件不变时, 衬底 温度 $T_{\mathrm{s}}$ 的变化对流体温度场的影响. 从 $700 \mathrm{~K}$ 等温
线的变化, 可以看到其由平直逐渐变化为隆起直至 陡直, 这表明随着基座温度的不断增高, 反应器内 衬底与 Showerhead 之间的温度梯度越来越陡峭, 热 浮力现象逐渐加剧.

在 MOCVD 反应器中, 对于特定的半导体材料 生长而言, 沉积温度主要与所需的沉积材料有关. 从经济上考虑, 需要一个相对高的生长速率, 所设 定的沉积温度要尽量高些; 然而, 较高的生长温度 很容易在外延层中引起材料的晶格缺陷. 所以, 在 保证适宜的反应温度下, 尽可能降低材料生长温度, 并将它限定在一个较窄的范围内, 以获取高质量的 外延材料.

当其他条件不变时, 计算了 3 种衬底温度对流 场分布的影响, 发现反应器内的流场分布的变化极 小, 3 种形态都与图 2(b)的区别甚微, 所以其计算图 形在此没有列出. 这表明在特定的设备结构尺寸和 一定的工艺条件下, 衬底温度的改变对流场的影响 也不显著.

\section{4 转速对热流场分布的影响}

MOCVD 反应器内部气体的流动, 不仅存在由 重力、密度差、压差引起的自然对流, 而且还存在基 座旋转引起的强制对流现象.

当其他条件不变时, 图 6 给出了基座转速为 80 , $100,120 \mathrm{r} / \mathrm{min}$ 时流场分布图. 同图 2(b)的流场分布 相比, 以下各图的流型有明显的改善, 在旋转的衬 底附近流线比较平直. 当转速在 $120 \mathrm{r} / \mathrm{min}$ 时, 在流
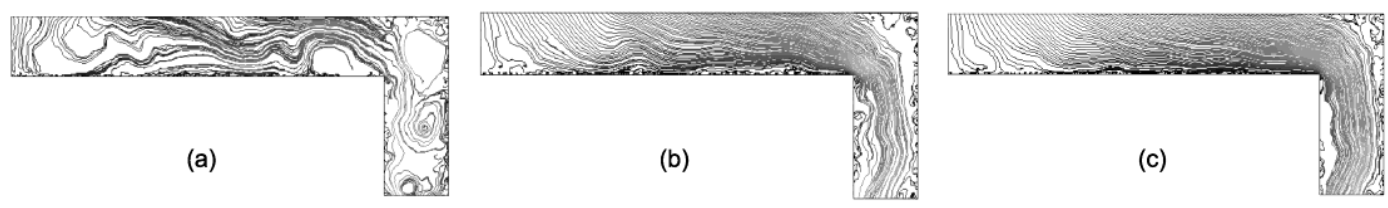

图 4 不同操作压力对流场结构的影响

(a) $P=101.3 \mathrm{kPa}$; (b) $P=13.3 \mathrm{kPa}$; (c) $P=6.66 \mathrm{kPa}$
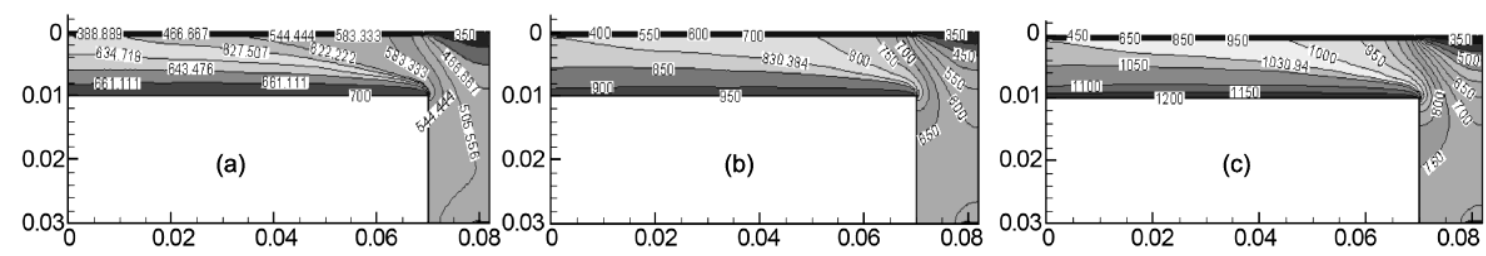

图 5 不同衬底温度对温度场分布的影响

(a) $T_{\mathrm{s}}=700 \mathrm{~K}$; (b) $T_{\mathrm{s}}=950 \mathrm{~K}$; (c) $T_{\mathrm{s}}=1200 \mathrm{~K}$ 

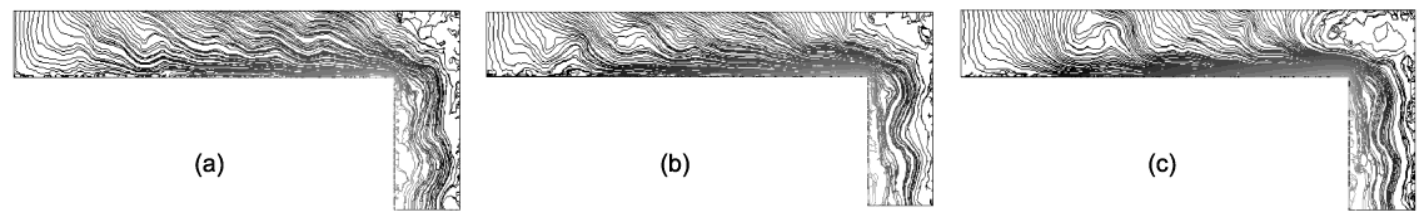

图 6 石墨舟转速对流场结构的影响

(a) $\omega=80 \mathrm{r} / \mathrm{min}$; (b) $\omega=100 \mathrm{r} / \mathrm{min}$; (c) $\omega=120 \mathrm{r} / \mathrm{min}$

道弯折处的旋涡增强变大, 并发展至基座边缘, 这 种流型对薄膜生长的均匀性将造成负面影响. 图 6 可 以看出, 增大基座转速, 具有“轴向抽吸”作用, 即能 够沿轴向向下强制拉动流动的气体, 使流线更贴近 了衬底表面, 形成平滑的滞止流，从而使气体反应 物在反应器内的分布更加合理; 基座旋转速度的增 大使离心驱动效应增强, 在“径向抽吸”的作用下, 反 应器内气体沿径向被排出, 使基座表面流线趋向平 直. 所以, 基座旋转的“厡浦效应”, 可以极大地改进 “记忆效应”产生的影响.

当其他条件不变时, 计算了基座旋转对温度场 分布的影响, 同没有旋转情况的图 3(b) 比较, 可以发 现 3 种转速的图 7 中, $808.4 \mathrm{~K}$ 等温线明显下移变得 更为平坦, 这表明旋转可以有效地改进主流道的温 度场分布, 使基座表面温度场的分布更趋合理, 这 样可以保证晶体生长的化学反应在相对稳定的条件 下进行, 有利于材料外延的均匀性.

总之，在不改变其它基准条件下，通过对图 6,7 的综合分析, 发现基座转速约为 $100 \mathrm{r} / \mathrm{min}$ 时, 不仅 可以获得稳定平直的流场分布, 即可较彻底地消除 “记忆效应”，增强传质效果即新鲜反应剂的更新和 反应产物的排出, 而且也有利于改善温度场分布的 均匀性. 所以, 当前绝大多数先进的 MOCVD 设备, 都采用旋转基座的方式, 以提高沉积材料生长的均 匀性.

\section{5 工艺参数优化条件下的热流场分布}

通过对 MOCVD反应器各关键参数进行数值运 算, 发现该设备的工艺条件如果维持在: 进气量为 $10 \sim 15 \mathrm{~L} \cdot \mathrm{min}^{-1}$ 、操作压力为6.66 26.7 $\mathrm{kPa}$ 、衬底温度 为880 1000 $\mathrm{K}$ 、转速为90 110 r.min的区间内, 均能 保持比较均匀的流场和温度场分布.

针对本文研究的生长设备与材料, 我们选 取一组适宜的工艺参数: 即反应器的进气量为 $12.5 \mathrm{~L} \cdot \mathrm{min}^{-1}$ 、操作压力为 $13.3 \mathrm{kPa}$ 、衬底温度为 $900 \mathrm{~K}$ 、转速为 $105 \mathrm{r} \cdot \mathrm{min}^{-1}$ 、内侧壁温度取为 $800 \mathrm{~K}$ 、 外侧壁温度为 $350 \mathrm{~K}$, 优化计算了反应器内部热 流场的分布状况.

从图8(a)的流场分析, 可以看到在反应器内流场 的分布比较均匀, 只是在流道的弯折处存在对流涡 旋，它对材料的外延质量影响不是很大; 此类流型 的产生, 是源于在轴向方向不断连续的进气流, 有 效压制了前一时刻喷人反应器内的气流径向方向流 动, 使之不能产生对流涡旋流动.

从图8(b)的温度场分析, 可以看到在反应器基座 上方等温线分布比较平直, 这说明通过合理调节 MOCVD反应器的重要工艺参数, 既可充分保证流体 流型的优良状态，又使温度场的分布得到了比较有 效的改善, 其结果十分有助于外延生长高质量的晶 体材料.
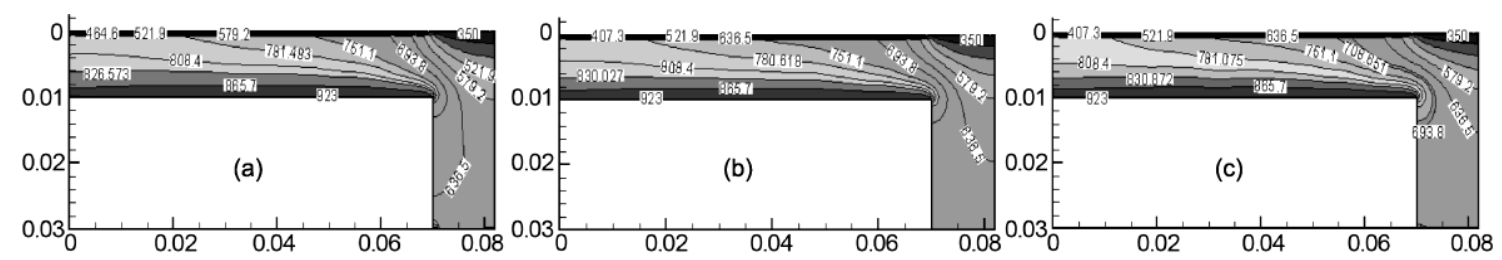

图 7 石墨舟转速对温度场分布的影响

(a) $\omega=80 \mathrm{r} / \mathrm{min}$; (b) $\omega=100 \mathrm{r} / \mathrm{min}$; (c) $\omega=120 \mathrm{r} / \mathrm{min}$ 

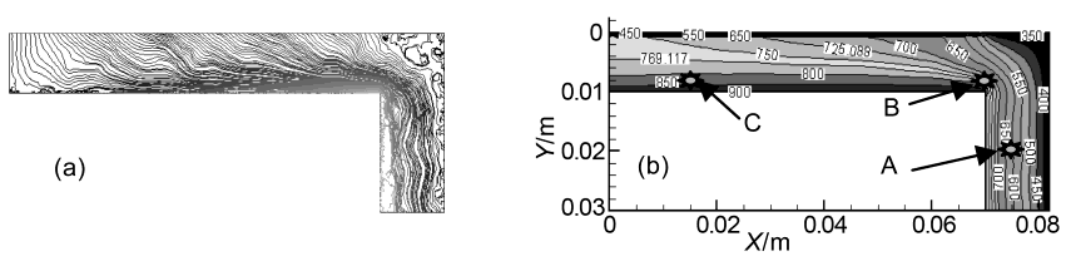

图 8 工艺参数优化条件下的热流场分布

\section{6 反应器中热流场策态行为的计算与分析}

通过对 MOCVD 反应器重要工艺控制参数进行 优化计算, 得到了稳态条件下优化的热流场分布, 其结果是进行瞬态行为研究的基础, 同时也可以将 其他影响因素降至最低, 这样才能凸显瞬态现象. 由于瞬态行为的强度比较微弱、持续的时间也较短, 任何物理测量技术都会对热流场的固有形态形成微 扰, 使其偏离原位而失真. 然而, 这正是 CFD (computational fluid dynamic)数值计算方法的优势所 在, 它可以充分揭示反应器内部流动过程中存在的 瞬态现象与规律.

为了便于计算 MOCVD 反应器内部流体的瞬态 流动特征, 选择反应器内 $\mathrm{A}(0.075,0.020), \mathrm{B}(0.070$, $0.008), C(0.015,0.008)$ 三点 (如图 8(b) 所示), 作 为考察其热流场稳定与否的图形化观测点. $\mathrm{A}$ 点着重 观测尾气流动情况, $\mathrm{B}$ 点透视基座边缘处衬底上方的 流动状态, $\mathrm{C}$ 点专注基座中部附近衬底上方的流动行 为, 通过对各点的数值计算揭示其内部流体流动的 规律. 具体操作是, 在保持其他优化工艺参数不变的 情况下, 对进气量进行阶跃微扰, 即 $Q$ 从 $12.5 \mathrm{~L} / \mathrm{min}$ 增 加为 $13.2 \mathrm{~L} / \mathrm{min}$ 时, 求解二维含时动力学方程组, 计 算了 MOCVD 热流场瞬态行为特征, 得到了直观、清 晰的结果.

微扰进气量对反应器内 $\mathrm{A}$ 点流场分布的影响, 可见图 9(a)所示: 初期流场的弛豫振荡剧烈, 后依次 递减; 经过约 $24 \mathrm{~s}$ 的时间后, 气体的流动进人较稳定 的自脉动振荡阶段. 由于存在随机湍流的作用, 自 脉动的周期性受到一定程度的干扰.

由图 9(b) 可见: 微扰进气量对反应器内温度场 分布的影响, 同流场响应行为不同的是, 温度场有 明显的延迟时间, 经过约 $38 \mathrm{~s}$ 后, 温度的变化逐渐进 人较稳定的状态, 亦进人自脉动阶段. 温度场与流 场的瞬态行为有别, 流场对微扰量的响应直接快捷, 而温度场则存在一定的延迟时间, 且驰豫振荡的时
间短、自脉动振荡周期幅度较大等特点. 这是由于反 应器系统内部, 流体的流动存在着随机脉动流、随机 热对流等因素, 所以造成温度场的自脉动现象比较 剧烈. 同时, 因为温度场的变化直接受气体流型的 支配与影响较大, 而且热系统主要依靠热对流的形 式传递能量, 故温度场的响应变化存在着一定的延 迟时间.

微扰进气量对反应器内 $\mathrm{B}$ 点流场分布的影响, 可见图 10(a)所示: 初期流场的弛豫振荡也较剧烈, 经过约 $25 \mathrm{~s}$ 的时间后, 气体的流动进入较稳定的自 脉动振荡阶段. 由图 10(b)可见: 微扰进气量对反应 器内温度场分布的影响, 计算发现其温度场也存在 明显的延迟时间, 经过约 $20 \mathrm{~s}$ 后, 温度的变化逐渐进 人较稳定的状态, 亦进人自脉动阶段. 由于 $\mathrm{B}$ 点附近 的流线最为密集, 流体内质点的相互作用最为强烈; 故同图 9(a)相比, B 点流场受到干扰的程度较为剧烈, 其自脉动的周期性因此更为不规则; 基于同样原因, 同图 9(b)相比, B 点温度场存在的延迟时间与弛豫振 荡的时间较短, 其自脉动振荡周期幅度受到极大地 抑制.

微扰进气量对反应器内 $\mathrm{C}$ 点流场分布的影响, 可见图 11(a)所示: 初期流场的弛豫振荡幅度较轻, 经过约 $20 \mathrm{~s}$ 的时间后, 气体的流动进人较稳定的自 脉动振荡阶段. 由图 11(b)可见: 微扰进气量对反应 器内温度场分布的影响, 经过约 $40 \mathrm{~s}$ 的延迟时间后, 温度场的变化进入较稳定的状态, 即进入自脉动阶 段.

由于 $\mathrm{C}$ 点附近的流线较为稀疏, 流体内质点的 相互作用与干扰程度不太强烈, 故同图 9(a), 图 10(a)相比, 其自脉动的周期性较明显; 基于同样 原因, $\mathrm{C}$ 点温度场对系统进气流量变化的反馈速度 较慢, 热对流传递能量的能力也较弱, 所以其温度 场的延迟时间更长，自脉动振荡周期的幅度也较 弱. 

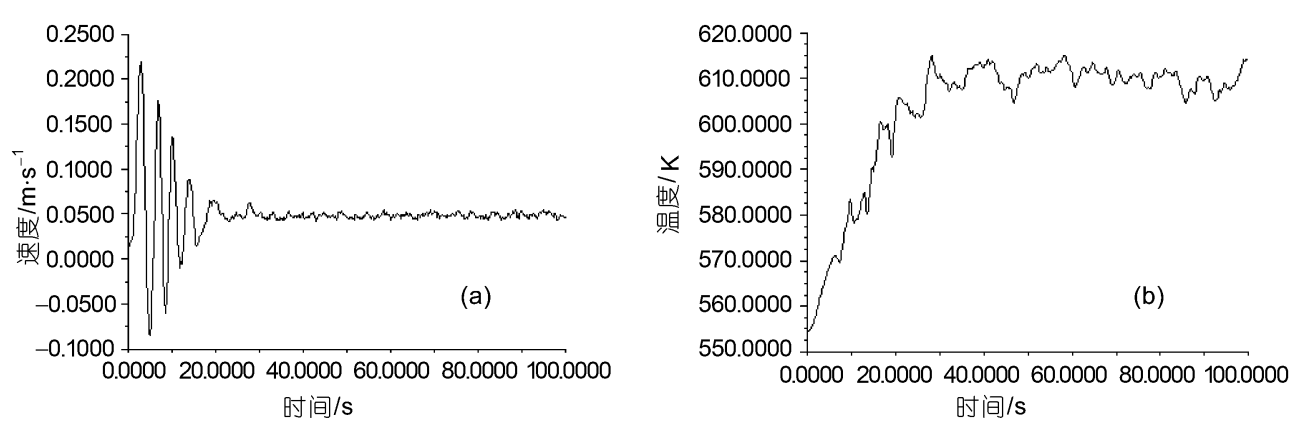

图 9 A 点热流场瞬态特征图
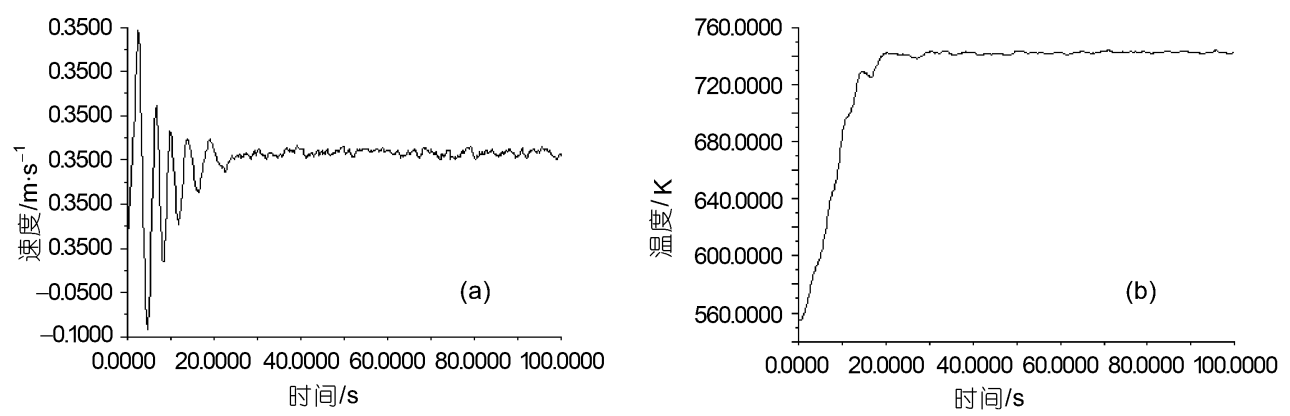

图 10 B 点热流场瞬态特征图
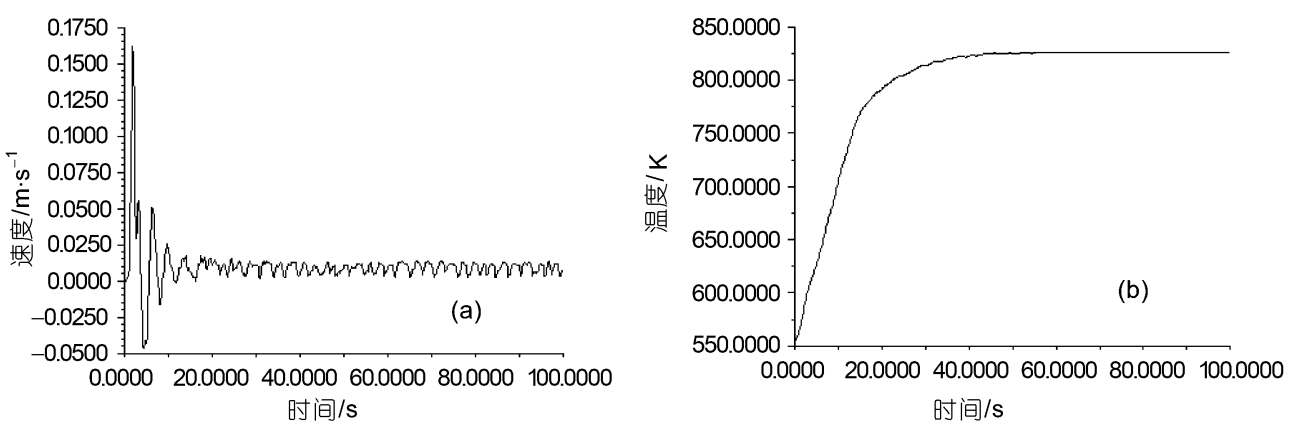

图 $11 \mathrm{C}$ 点热流场瞬态特征图

从衬底上方流体运动的瞬态行为分析, 可以发 现流场自脉动振荡的积极意义是：可以增强衬底表 面的传质效果, 有利于新鲜反应物向衬底方向的扩 散, 从而增加了晶体生长的速度; 当然, 自脉动振荡 现象也存在消极作用, 即对晶体材料外延层间的均 匀性、各界面间的陡峭分布, 以及批量制备晶体材料 的一致性等方面, 存在不利的影响.

由于 MOCVD 生长设备具有规模大、成本低、 性能可靠等特点, 它已经成为商业上制备化合物半 导体材料的首选设备. 然而, MOCVD 反应器内部气 体实际流动现象十分复杂, 既包含了复杂的随机脉 动流动, 以及由随机因素引发的不确定热流场的不
规则变化; 同时, 包含了传热、传质和化学反应, 以 及它们之间的相互耦合作用. 所以, 使用 MOCVD 设 备, 外延生长出原子级陡峭异质界面的难度比较大.

随着半导体材料与器件的功能越来越强大, 应 用的领域越来越广阔; 特别是近年来, 基于量子点、 量子线、量子阱超晶格等高端的纳米材料与器件的 出现, 加上横向外延技术、低温缓冲层技术、组分渐 变缓冲层技术, 以及光电系统集成等复杂技术的综 合运用, 就要求商业级的 MOCVD 设备生长出更高 质量的异质结构材料, 即在衬底上方外延出特定图 形、特定组分、特定厚度、特定导电类型、特定电学 和光学性能的晶体材料, 这就需严格控制 MOCVD 设 
备的工艺生长条件, 其中较重要的是, 要对瞬态现象 加以足够的重视与研究, 严密地设计与控制周期性 地切换前驱物进人反应器, 尽快使反应物在衬底上 方形成稳定的流动状态, 只有这样才可能较有效地 得到原子级陡峭的异质界面分布. 所以, 瞬态现象 的研究, 为解决上述问题与难点, 以及实现材料批 量生长的可重复性等外延特征与规律, 有着非常重 要的意义. 总之, 通过运用 CFD 数值计算技术, 同时 采用微扰进气量分析法, 可以直观剖析反应器内部 流体流动的瞬态行为, 这对研究反应器内流体因组 分切换时产生的延迟时间、弛豫振荡和自脉动振荡
等随机现象大有益处.

\section{3 结论}

本文对 MOCVD 设备的重要工艺参数进行了较 为详细的研究, 从如何获得较佳的热流场的分布出 发, 在此基础上考察了反应器的瞬态行为变化, 分 析了瞬态现象产生和变化的原因, 其结论将有助于 深化理解 MOCVD 反应器中流体的输运过程与工艺 参数的内在关系, 这对提高外延层的均匀性、界面的 陡峭性和生长的可重复性有较积极的意义, 为难度 较大的“异系异质兼容”等复杂外延生长技术, 奠定 了坚实的研究基础.

\section{参考文献}

1 Manasevit H M. Single-crystal gallium arsenide on insulating substrates. Appl Phys Lett, 1968, 12: 156-159[doi]

2 Dupuis R D. Metalorganic chemical vapor deposition of III - V semiconductors. Science, 1984, 226: 623-629[doi]

3 Ponce F A, Bour D P. Nitride-based semiconductors for blue and green light-emitting devices. Nature, 1997, 386: 351—359[doi]

4 Kuykendall T, Ulrich P, Ulrich P, et al. Complete composition tunability of InGaN nanowires using a combinatorial approach. Nat Mater, 2007, 6: 951-956[doi]

5 Hostein R, Michon A, Beaudoin G, et al. Time-resolved characterization of InAsP/InP quantum dots emitting in the C-band telecommunication window. Appl Phys Lett, 2008, 93: 073106 [doi]

6 Wang X L, Wang C M, Hu G X, et al. Growth and characterization of 0.8 - $\mu \mathrm{m}$ gate length AlGaN/GaN HEMTs on sapphire substrates. Sci China Ser F-Inf Sci, 2005, 48: 808-814 [1doi]

7 Wang C A, Groves S H, Palmateer S C. Flow visualization studies for optimization of OMVPE reactor design. J Cryst Growth, 1986, 77: $136-143$ [doi]

8 Stock L, Richter W. Vertical versus horizontal reactor: An optical study of the gas phase in a MOCVD reactor. J Cryst Growth, 1986, 77: $144-150$ [doi]

9 Nami Z, Erbil A, May G S. Reactor design considerations for MOCVD growth of thin films. IEEE Trans Semicond Manuf, 1997, 10: $295-306 \underline{\text { [doi] }}$

10 陈俊, 张书明, 张宝顺, 等. 缓冲层生长压力对 MOCVD GaN 性能的影响. 中国科学 E 辑: 技术科学, 2004, 34: 58一 63

11 徐现刚, 崔得良, 唐詰, 等. InGaAs/InP 异质结界面层的应变研究. 中国科学 A 辑, 2001, 31: 817-822

12 张佳文, 高鸿楷, 张济康, 等. MOCVD 过程中回流现象的数值模拟. 半导体学报, 1994, 15: 268-272

13 左然, 张红, 刘祥林. 径向三重流 MOCVD 反应器输运过程的数值模拟研究. 半导体学报, 2005, 26: 977一 982

14 刘奕, 陈海昕, 符松. GaN-MOCVD 设备反应室流场的 CFD 数值仿真. 半导体学报, 2004, 25: 1639-1645

15 郭文平, 郡嘉平, 罗毅, 等. MOCVD 生长 GaN 材料的模拟. 半导体学报, 2005, 26: 735-739

16 杨云柯, 高立华, 陈海昕, 等. 喷淋式 GaN-MOCVD 反应室的 CFD 数值仿真及优化. 力学学报, 2007, 24: 173-178

17 Mitrovic B, Gurary A, Quinn W. Process conditions optimization for the maximum deposition rate and uniformity in vertical rotating disc MOCVD reactors based on CFD modeling. J Cryst Growth, 2007, 303: 323-329 [doi]

18 Martin C, Dauelsberg M, Protzmann H, et al. Modeling of group- III nitride MOVPE in close coupled showerhead reactor and planetary reactor. J Cryst Growth, 2007, 303: 318-322[doi]

19 钟树泉, 任晓敏, 王琦, 等. MOCVD 反应器热流场的数值模拟研究. 人工晶体学报, 2008, 37: 1342一1348

20 Zhong S Q, Ren X M, Huang Y Q, et al. Analysis and simulation of process parameters for epitaxy of InP-based compound semiconductor materials. APOC Proc SPIE, 2008, 7135: 71353M 